\title{
Subgroups of finitely presented groups with solvable conjugacy problem
}

\author{
A.Yu.Olshanskii and M.V. Sapir*
}

\begin{abstract}
We prove that every countable group with solvable power problem embeds into a finitely presented 2-generated group with solvable power and conjugacy problems.
\end{abstract}

\section{Introduction}

We say that a group $G$ is recursively presented if $G=\left\langle x_{1}, x_{2}, \ldots \mid R\right\rangle$ where $R$ is a recursive set of words. In that case the set of all equalities $u=1$ where $u$ is a word in $x_{1}, x_{2}, \ldots$, that are true in $G$ is recursively enumerable. Recursive presentability follows from solvability of the word problem. Note that in this paper, we always consider countable groups together with their presentation: a countable group may have a recursive presentation and a non-recursive one.

Let $G$ be a recursively presented (but not necessarily finitely generated) group. We shall say that $G$ has solvable power problem if there exists an algorithm which, given $u, v$ in $G$ says if $v=u^{n}$ for some $n \neq 0$. Notice that solvability of power problem implies solvability of the word problem (take $u=1$ ). The converse implication does not hold (see [McC2] or [Col2]). Notice also that if $G$ has solvable power problem then it has solvable order problem that is there exists an algorithm that given $u \in G$ computes the order of $u$ in $G$. Indeed, one can first find out if there exists an $n \neq 0$ such that $1=u^{n}$. Then if such an $n$ exists, find the smallest such $n$ using an algorithm that solves the word problem in $G$. Notice that the "classical" formulation of the power problem does not exclude the case $n=0$, but that change is not significant since the case $n=0$ is a particular case of "our" power problem for $v=1$.

In [KT] (Problem 5.21) Collins asked whether every torsion-free group with solvable word problem can be embedded into a finitely presented group with solvable conjugacy problem.

In this paper, we shall give positive answer to Collins' question under the stronger assumption of solvability of the power problem. Adding that restriction allowed us to drop the "torsion-free" restriction from Collins' problem.

Theorem 1. Every countable group with solvable power problem is embeddable into a 2-generated finitely presented group with solvable conjugacy and power problems.

Thus embedding a group into a finitely presented group can dramatically improve its algorithmic properties.

\footnotetext{
*2000 Mathematics Subject Classification: 20E07, 20F06, 20F10

* Both authors were supported in part by the NSF grant DMS 0072307. In addition, the research of the first author was supported in part by the Russian Fund for Basic Research 02-01-00170, the research of the second author was supported in part by the NSF grant DMS 9978802 and the US-Israeli BSF grant 1999298.
} 
Remark 2. Notice that solvability of power problem cannot be replaced in Theorem 1 by solvability of word problem. Indeed, there exists an example of a group with solvable word problem that cannot be embedded into a group with solvable conjugacy problem (this example is attributed by Collins to Macintyre in [KT, Problem 5.21]).

We also prove the following theorem that generalizes the main result of OS2 and gives a positive answer to Collins' problem 5.22 from $\underline{\mathrm{KT}}$.

Theorem 3. Every (countable) group with solvable conjugacy problem can be embedded into a 2-generated finitely presented group with solvable conjugacy problem.

In OS2, we proved Theorem 3 only for finitely generated groups.

Remark 4. One can try to prove that every countable torsion-free group $G$ with solvable word problem is embeddable into a finitely presented group with solvable word problem (and solve Collins' problem 5.21 from $\left[\mathrm{KT}\right.$ ) as follows. First embed $G$ into a group $G^{\prime}$ where all non-trivial elements are conjugate using HNN extensions as in LS. Then use Theorem 3 to embed $G^{\prime}$ into a finitely presented group with solvable conjugacy problem. Unfortunately this idea does not work: the group $G^{\prime}$ would not necessarily have solvable word problem. Indeed, an HNN extension of a group has solvable word problem only if the group has solvable membership problems for the associated subgroups. This is the reason why we cannot avoid the solvability of power problem in Theorem 1 .

\section{Proofs}

As usual, we are going to use van Kampen diagrams to represent deduction of relations in groups. Throughout the paper, for every van Kampen diagram $\Delta, \partial \Delta$ denotes its boundary, and for every path $p$ in a van Kampen diagram, $\phi(p)$ denotes its label.

The following lemma is proved in Col1.

Lemma 5. Let $G$ be a recursively presented group with solvable power problem. Let $a, b$ be two elements in $G$ of the same order. Then the HNN extension $G_{a, b}=\langle G, t| t^{-1}$ at $\left.=b\right\rangle$ has solvable power problem.

In order to embed countable groups into 2-generated groups we use a set of positive words in the alphabet $\{a, b\}$ which is similar to the sets used for similar purposes in [McC1], [LS] and O1:

$$
A_{i}=a^{100} b^{i} a^{101} b^{i} \ldots a^{199} b^{i}, \quad i=1,2, \ldots
$$

We denote by $H$ the subgroup generated by these words in the free group $F(a, b)$. The reduced words in $F(a, b)$ representing elements of $H$ are called $H$-words. A cyclic $H$-word is a cyclically reduced word that is freely conjugate to an $H$-word in $F(a, b)$. Set $\lambda=\frac{1}{11}$.

Lemma 6. (1) Let $U V^{\prime}$ and $U V^{\prime \prime}$ be two distinct cyclic $H$-words. Then either

$$
|U|<\lambda \min \left(\left|U V^{\prime}\right|,\left|U V^{\prime \prime}\right|\right)
$$

or the word $V^{\prime}\left(V^{\prime \prime}\right)^{-1}$ is a free conjugate in $F(a, b)$ of an $H$-word.

(2) The set of (cyclic) $H$-words and the set of their subwords are recursive.

(3) Suppose that a cyclic $H$-word $W$ has prefixes $w_{1}$ and $w_{2} w_{1}$ for some $\left|w_{1}\right| \geq \lambda|W|$. Then $w_{2}$ is a cyclic $H$-word. 
Proof. (1) This statement was proved in [01] for $\lambda=\frac{1}{7}$. The same proof works for $\lambda=\frac{1}{11}$ since the factor $\frac{1}{30}$ can be replaced by $\frac{1}{50}$ in Lemma 1 of 01 .

(2) This follows from the explicit forms (11) for the generators of the subgroup $H$.

(3) This also follows from the definition (11) and the small cancellation property (1).

Consider the following construction (cf. [O1]) of an embedding of countable groups into finitely generated groups. Let $G=\left\langle x_{1}, x_{2}, \ldots \mid R\right\rangle$ be a group. Without loss of generality we shall assume that $R$ consists of all non-empty relations of the group $G$. Denote by $\bar{R}$ be the set of words in the alphabet $\{a, b\}$ obtained by substituting $A_{i}$ for $x_{i}$ in every word from $R$. We shall denote the group $\langle a, b \mid \bar{R}\rangle$ by $\bar{G}$.

We shall prove (Lemma 8 below) that $G$ embeds into $\bar{G}$ and that this embedding preserves solvability of power and conjugacy problems (Lemmas 10 and 11] below). Note that in the literature, there exist embeddings of countable groups into finitely generated groups which preserve solvability of either power problem ([McC1] ) or conjugacy problem (Col1]). We need to preserve solvability of both power and conjugacy problems so formally we cannot use embeddings from either [McC1 or [Col1. Besides, our construction is easier and it yields 2-generated groups while constructions from $\mathrm{McC1}$ and Col1 give 3- and 4-generated groups respectively.

The following statement is obvious.

Lemma 7. The group $\bar{G}$ is finitely presented provided $G$ is finitely presented.

A (disc or annular) van Kampen diagram over $\bar{R}$ will be called minimal if it contains the minimal possible number of cells among all diagrams with the same boundary labels.

For every $\alpha>0$, a cell $\pi$ in a disc or annular diagram $\Delta$ is called a Greendlinger $\alpha$-cell if $\partial \pi$ contains a subpath $p$ with $|p| \geq \alpha|\partial \pi|$, and $p$ is a subpath of a boundary component of $\Delta$. The path $p$ will be called a Greendlinger $\alpha$-path of $\pi$.

We say that a diagram or a map $\Delta$ satisfies the small cancellation condition $C^{\prime}(\lambda)$ if for every two cells $\pi, \pi^{\prime}$ in $\Delta$ (possibly $\pi=\pi^{\prime}$ ), and every common subpath $p$ of $\partial \pi$ and $\left(\partial \pi^{\prime}\right)^{-1}$, we have $|p|<\lambda \min \left(|\partial \pi|,\left|\partial \pi^{\prime}\right|\right)$.

If $\pi=\pi^{\prime}, p$ is a common subpath of $\partial \pi$ and $(\partial \pi)^{-1},|p| \geq \lambda \min \left(|\partial \pi|,\left|\partial \pi^{\prime}\right|\right)$, and $\partial \pi=$ $q^{\prime} p q^{\prime \prime} p^{-1}$, where $q^{\prime}$ surrounds the hole of the annular diagram $\Delta$, then $\pi$ is said to be a hoop.

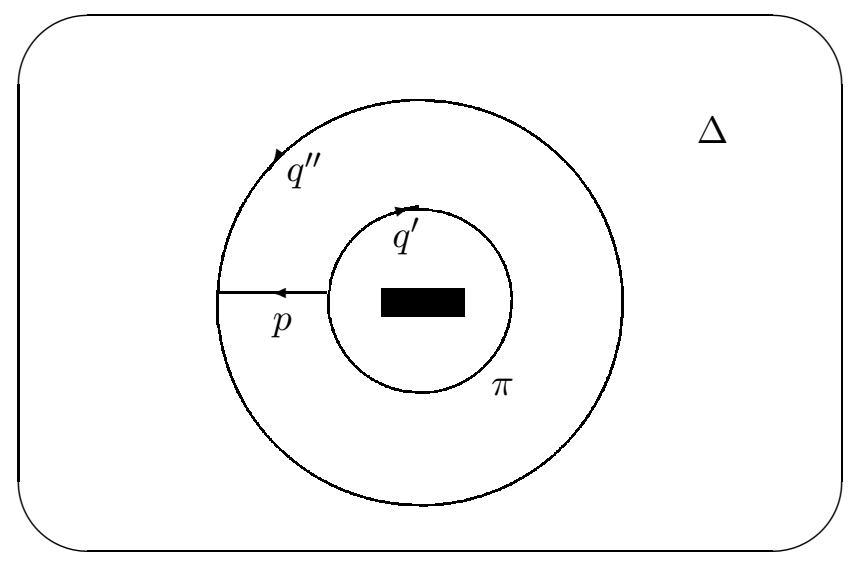

Figure 1: A hoop.

Lemma 8. (1) A minimal disc or annular diagram over $\bar{R}$, having no hoops in annular case, satisfies the $C^{\prime}(\lambda)$-condition. 
(2) The labels of the subpaths $q^{\prime}$ and $q^{\prime \prime}$ in the boundary of a hoop (see the definition and notation before the lemma) are cyclic $H$-words.

(3) If a boundary component $q$ of a diagram (annular diagram) over $\bar{R}$ is an $H$-word (a cyclic $H$-word), and a cell $\pi$ has boundary $p_{1} p_{2}$, where $q=p_{1} q^{\prime}$ and $\left|p_{1}\right| \geq \lambda|\partial \pi|$, then the label of the path $p_{2}^{-1} q^{\prime}$ is freely equal (freely conjugate) to an $H$-word.

(4) If a disc map $\Delta$ satisfies $C^{\prime}(\lambda)$-condition and contains a cell, then (a) it has a Greendlinger $(1-3 \lambda)$-cell, and (b) the number of (non-directed) edges of $\Delta$ does not exceed $\left(1+3 \lambda(1-6 \lambda)^{-1}\right)|\partial \Delta|$.

(5) If an annular map $\Delta$ contains at least one cell and satisfies $C^{\prime}(\lambda)$-condition, then either $\Delta$ has a Greendlinger $(1-4 \lambda)$-cell or every cell $\pi$ of $\Delta$ has boundary subpaths $p_{1}$ and $p_{2}$ on both boundary components; in particular $\left|p_{1}\right|+\left|p_{2}\right|>(1-2 \lambda)|\partial \pi|$.

(6)(a) The mapping $x_{i} \rightarrow A_{i}$ extends to an embedding of the group $G$ into $\bar{G}$. (b) Under this embedding, two elements of $G$ that are conjugate in $\bar{G}$ are also conjugate in $G$.

Proof. Lemma [ implies assertions (1), (2), (3) and (6)(a) as this was shown in $\S 2$ of [01. The proof of $(6)(\mathrm{b})$ is similar to the proof of (6)(a) but one should take an annular diagram instead of a disc one and use assertion (2) when considering annular subdiagrams between two hoops. (The statement (6)(b) was also proved by Ilya Belyaev in Bel. .) The proof of assertion (4)(a) can be found in [LS] (see Theorem 4.4, formulated in terms of relations). The assertion (4)(b) follows from (4)(a) by straightforward induction on the number of cells in $\Delta$. The assertion (5) is contained in LS, Theorems 5.3 and 5.5]. (In the proofs of these theorems, one can replace $C^{\prime}(1 / 6)$ by $C^{\prime}(\lambda)$, where $\lambda \leq 1 / 6$. Accordingly one can replace $(1 / 2) R$ by $(1-3 \lambda) R$ in the assumptions of these theorems. Since $i(D)=p / q+2=4$ in the formulation of [LS, Theorem 5.3], $D$ is a Greendlinger $(1-4 \lambda)$-cell.)

We call a (cyclically) reduced word $w$ (cyclically) $\bar{R}$-reduced if it has no (cyclic) subword $v$, where $v$ is a subword of a relator $r \in \bar{R}$ and $|v|>\frac{1}{2}|r|$. If the word problem is decidable for $G$, then, by Lemma $6(2)$, for every word $w$, one can effectively find a (cyclically) $\bar{R}$-reduced word $w^{\prime}$ which is equal (or is conjugate) to $w$ in the group $\bar{G}$.

Lemma 9. Assume that a $\bar{R}$-reduced (cyclically $\bar{R}$-reduced) word $w$ is equal (is conjugate) in $\bar{G}$ to an $\mathrm{H}$-word (a cyclic $\mathrm{H}$-word). Then $w$ is equal (is conjugate) to an $\mathrm{H}$-word (a cyclic $\mathrm{H}$-word) in the free group $F(a, b)$.

Proof. We consider only the cyclic case. Let $\Delta$ be a minimal diagram for the conjugation of $w$ and some cyclic $H$-word $w^{\prime}$, and let $\Delta$ have minimal number of cells over all such $w^{\prime}$. By Lemma 8(2), $\Delta$ has no hoops. If $\Delta$ has a cell $\pi$, then, by Lemma 8 (3) and by the minimality of $\Delta, \pi$ cannot have a common boundary subpath of length at least $\lambda|\partial \pi|$ with the contour of $\Delta$ labeled by $w^{\prime}$. Then, by Lemma $8(2),(5), \pi$ must have a boundary subpath of length greater than $(1-4 \lambda)|\partial \pi|$, lying on the contour of $\Delta$ labeled by $w^{\prime}$. This contradicts the cyclic $\bar{R}$-reducibility of $w$, since $\lambda \leq 1 / 8$.

Lemma 10. If the group $G$ has decidable word or conjugacy problem, then so has the group $\bar{G}$.

Proof. Here we consider only the conjugacy problem. Let $u$ and $v$ be two words under our investigation. We may assume that they are cyclically $\bar{R}$-reduced since the conjugacy (and the word) problem is decidable for $G$. By Lemma 9, we can also assume that they are $H$-words if they are conjugate to $H$-words in $\bar{G}$. In the later case, it suffices to check conjugacy of $u$ and $v$ in $G$ by Lemma 8 (6)(b). If $u$ and $v$ are conjugate in $\bar{G}$, but none of them is conjugate to a cyclic $H$-word, then a minimal diagram $\Delta$ for the conjugation of $u$ and $v$ has no hoops by Lemma 8 
(2). It also has no Greendlinger $\alpha$-cells for $\alpha>1 / 2$, since $u$ and $v$ are cyclically $\bar{R}$-reduced. It follows from Lemma 8 , part (5), that the sum of perimeters of the cells of $\Delta$ does not exceed $\left(1-2 \lambda^{-1}\right)(|u|+|v|)$, and therefore such diagrams can be checked by exhaustion.

Lemma 11. Let $G=\langle X \mid R\rangle$ be a recursively presented group with solvable power problem. Then the group $\bar{G}$ has solvable power problem.

Proof. Let $u$ and $v$ be words in the alphabet $\{a, b\}$. Suppose that $v=u^{n}$ in $\bar{G}$, where $n \geq 1$. By Lemmas [ (2) and 9] we may assume that the word $u$ is cyclically $\bar{R}$-reduced and it is a cyclic $H$-word if it is a conjugate of an $H$-word in $\bar{G}$. If $u$ is an $H$-word, then so is $v$, and, by Lemma 8 (6)(a), we may refer to the solvability of the power problem in $G$. Therefore we further assume that $u$ is not a conjugate of an $H$-word in $\bar{G}$.

Let $\Delta$ be a minimal diagram over $\bar{R}$ whose contour is $q_{1} q_{2}$, where $q_{1}$ and $q_{2}$ are labeled by $u^{n}$ and $v^{-1}$, respectively. Call a cell $\pi$ of $\Delta$ suitable, if its boundary has a common subpath $p$ with $q_{1}$, and $|p|>(1 / 2+\lambda)|\partial \pi|$.

Suppose $\Delta$ has a suitable cell $\pi$. If $|p| \geq|u|+\lambda|\partial \pi|$, then $u$ is a cyclic $H$-word by Lemma 6 (3), since the label of $p$ is a subword of $u^{n}$; a contradiction. But it follows from the inequality $|p|<|u|+\lambda|\partial \pi|$ that $|p|<|u|+\lambda(1 / 2+\lambda)^{-1}|p|$, i.e. $|p|<\left(1-\lambda(1 / 2+\lambda)^{-1}\right)^{-1}|u|$. It also follows that the word $u$ is not cyclically $\bar{R}$-reduced, since an application to a cyclic permutation of $u$ of the $\bar{R}$-relator corresponding to $\pi$, gives a word of length at most

$$
|\partial \pi|-|p|+\lambda|\partial \pi|<\frac{1}{2}|\partial \pi| \leq \frac{1}{2}(1 / 2+\lambda)^{-1}|p|<(1+2 \lambda)^{-1}\left(1-\lambda(1 / 2+\lambda)^{-1}\right)^{-1}|u|=|u|
$$

The contradiction shows that $\Delta$ has no suitable cells.

Now assume that $\Delta$ has a cell $\Pi$ having two maximal boundary subpaths $p_{1}$ and $p_{2}$ on $q_{1}$ (a "bad" cell). Then there must be cells in the subdiagram $\Gamma$ between $\Pi$ and $q_{1}$, and one may chose $\Pi$ so that there are no bad cells in $\Gamma$. Similarly we assume that there are no cells in $\Gamma$ having two maximal boundary subpaths on $\partial \Pi$, since otherwise we can decrease the number of cells in $\Gamma$. Then by Lemma 8 (1), (4)(a), the diagram $\Delta$ has a suitable cell inside $\Gamma$ since $1-3 \gamma-\lambda \geq 1 / 2+\lambda$.

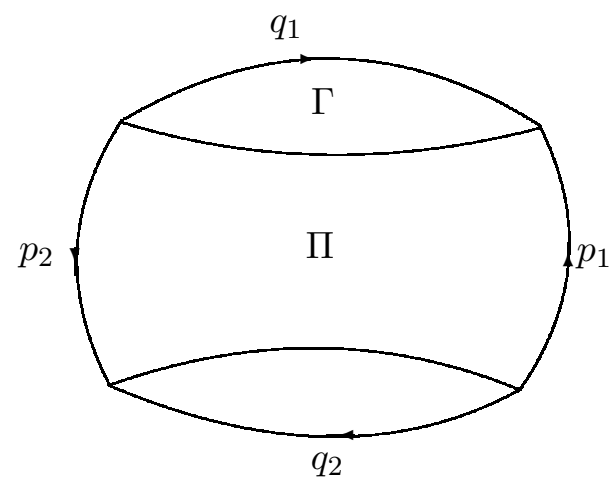

Figure 2: A "bad" cell.

This contradiction shows that there are no bad cells in $\Delta$. The same consideration shows that the path $q_{1}$ is simple. Then $\Delta$ has at least $(1 / 2+\lambda)^{-1}\left(\left|q_{1}\right|-\left|q_{2}\right|\right)$ edges because it has no suitable cells. This inequality and Lemma 8 (4)(b) give a linear upper bound for $\left|q_{1}\right|$ in terms of $\left|q_{2}\right|$ since $(1 / 2+\lambda)^{-1}>1+3 \lambda(1-6 \lambda)^{-1}$. This reduces the problem to the word problem in $\bar{G}$ which is decidable by Lemma 10 . 
Lemma 12. Let $G$ be a finitely generated recursively presented group with solvable power and conjugacy problems. Then the group $G$ can be embedded into a finitely presented group $H$ with solvable power and conjugacy problems.

Proof. Let us use the embedding from OS2. We are going to use the notation and results from OS2. In particular, from now on we shall denote $G$ by $\mathcal{G}$, and $H$ by $\mathcal{H}$ as in OS2. We have proved in OS2 that $\mathcal{H}$ has solvable conjugacy problem. It remains to prove the solvability of the power problem.

Recall that the set of generators of $\mathcal{H}$ consists of $k$-letters, $a$-letters, $\theta$-letters and $x$-letters. The subgroup $\mathcal{G}$ in $\mathcal{H}$ is generated by a subset $\mathcal{A}\left(P_{1}\right)$ of the set of $a$-letters. As in OS2, we include all relations of $\mathcal{G}$ into the presentation of $\mathcal{H}$.

Among the relations of $\mathcal{H}$, there is one, called the $h u b$ which is a word in $k$-letters of length $N$ (in OS2 $N$ is any even number $\geq 8$; here we take $N \geq 14$ ), all letters occurring in the hub are different. Every non-hub cell in a van Kampen diagram over $\mathcal{H}$ that contains an edge labeled by a $k$-letter $K$ (i.e. a $k$-edge) also contains an edge labeled by $K^{-1}$, so we can consider $k$-bands in $\Delta$ (see the precise definition of a band in OS2 ). Similarly, we can consider $\theta$-bands and $a$-bands. The group given by the presentation of $\mathcal{H}$ without the hub is denoted by $\mathcal{H}_{1}$.

1. Let $u$ be a word in generators of $\mathcal{H}$. Consider a word $u^{\prime}$ that is a conjugate of $u$ in $\mathcal{H}$ and has minimal number of $k$-letters among all words in the conjugacy class of $\mathcal{H}$. Consider a minimal annular diagram $\Delta$ for this conjugation with contours $p$ and $p^{\prime}, \phi(p)=u, \phi\left(p^{\prime}\right)=u^{\prime}$. By the minimality in the choice of $u^{\prime}$, every hub $\Pi$ of $\Delta$ has at most $N / 2 k$-bands starting on $\Pi$ and ending on $p^{\prime}$. Assume there exists a hub in $\Delta$. Since $N \geq 10$, OS2, Lemmas 10.4 and 10.3] provide us with a hub $\Pi$ connected with $p$ by two $k$-bands, such that there are no hubs between these $k$-bands. By OS2, Lemma 10.5], the hub $\Pi$ can be effectively cut out of $\Delta$ with a recursive replacement of $u$ by a conjugate word. However, by [OS2, Lemmas 10.4 and 10.3], the number of hubs in $\Delta$ is not greater than the doubled number of $k$-letters in $u$. Therefore we can recursively obtain a word $u^{\prime \prime}$, such that $u^{\prime \prime}$ and $u^{\prime}$ are conjugate in the group $\mathcal{H}_{1}$. Now, by OS2, Lemma 5.6], starting with $u^{\prime \prime}$, we can recursively obtain an $\mathcal{H}_{1}$-conjugate word $u^{\prime \prime \prime}$ having minimal number of $k$-letters in its $\mathcal{H}$-conjugacy class and minimal number of $\theta$-letters in its $\mathcal{H}_{1^{-}}$ conjugacy class. By OS2, Lemma 5.6], $u^{\prime \prime \prime}$ is also not conjugate to a word with fewer $a$-letters if a deduction of the latest conjugation does not employ $\theta$-relations. We shall call a word $u^{\prime \prime \prime}$ with these three properties cyclically minimal. Similarly for every word $w$ in the generators of $\mathcal{H}$, we can effectively find a word $w^{\prime}$ which is equal to $w$ in $\mathcal{H}$ and has minimal number of $k$-letters among all words that are equal to $w$ in $\mathcal{H}$, minimal number of $\theta$-letters among all words that are equal to $w^{\prime}$ in $\mathcal{H}_{1}$, and not equal in $\mathcal{H}_{1}$ to a word with fewer $a$-letters if a deduction of this equality does not use $\theta$-relations. Such words $w^{\prime}$ will be called minimal.

2. Let $u, w$ be words in the generators of $\mathcal{H}$. Suppose that $w=u^{n}$ in $\mathcal{H}$ for some $n \geq 0$. To prove the lemma, we need to recursively bound $n$ in terms of $w$ and $u$. We can assume that the word $u$ is cyclically reduced and cyclically minimal by part 1 . We can also assume that $w$ is minimal. Consider the corresponding minimal van Kampen diagram $\Delta$ such that $\partial \Delta=p q^{-1}$, $\phi(p)=w, \phi(q)=u^{n}$.

3. We set $N^{\prime}=N / 2+1$. Suppose that there exists a hub $\pi$ in $\Delta$ such that some consecutive $k$-bands $\mathcal{B}_{1}, \ldots, \mathcal{B}_{N^{\prime}}$ starting on $\partial \pi$ end on $q$, and between two consecutive $k$-bands starting on $\partial \pi$, there are no other $k$-bands. We shall call these hubs $u$-close. In particular, it implies that between any two consecutive bands $\mathcal{B}_{i}$, there are no hubs (since $k$-bands do not intersect). Since all $k$-edges on $\partial \pi$ have different labels, and there are no other $k$-edges between the end edges of $\mathcal{B}_{1}, \ldots, \mathcal{B}_{N^{\prime}}$ on $q$, we can conclude that all the bands $\mathcal{B}_{1}, \ldots, \mathcal{B}_{N^{\prime}}$ connect $\partial \pi$ with a subpath of $q$ labeled by a cyclic shift of $u$, contrary to the assumption that $u$ is minimal, because 
$N^{\prime}>N-N^{\prime}$. Hence $\Delta$ contains no $u$-close hubs. Since $N \geq 14$, this result, OS2, Lemma 10.4] and [02, Lemma 3.4] give a linear upper bound for the number of hubs of $\Delta$ in terms of $|w|$.

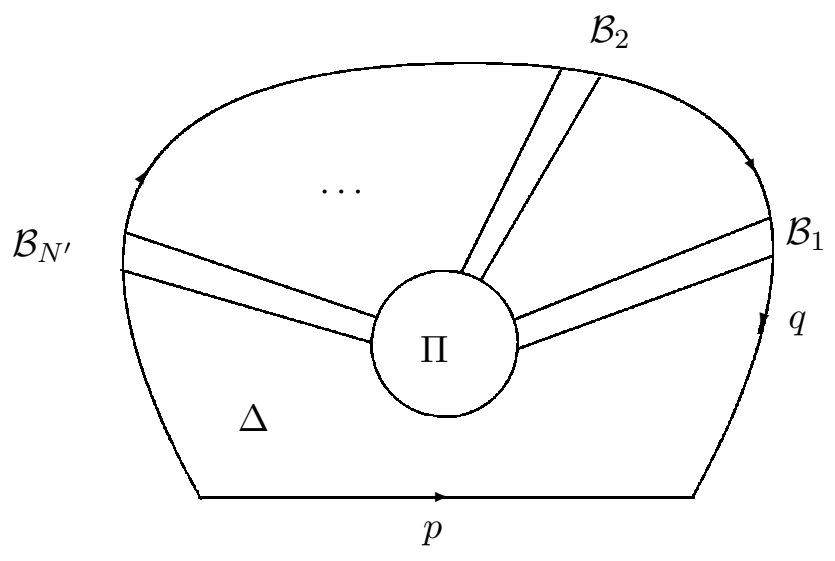

Figure 3: u-close hub.

4. Suppose that there are two $k$-edges $e, e^{\prime}$ in $q$ which are connected by a $k$-band $\mathcal{B}$. Consider the subdiagram $\Delta^{\prime}$ bounded by $\mathcal{B}$ and $q\left(\Delta^{\prime}\right.$ does not contain $\left.\mathcal{B}\right)$. We can assume that $\Delta^{\prime}$ does not contain $k$-bands connecting two edges on $q \cup \partial \Delta^{\prime}$. If there was a hub in $\Delta^{\prime}$ then by OS2, Lemmas 10.4 and 10.3] there would be a $u$-close hub there. Hence by part 1 there are no hubs in $\Delta^{\prime}$. Hence there are no $k$-edges between $e$ and $e^{\prime}$ in $q$, therefore $e$ and $e^{\prime}$ belong to a subpath of $q$ labeled by a cyclic shift $u_{1}$ of $u$. The word that labels the subpath of $q$ starting at $e$ and ending at $e^{\prime}$ is equal in $\mathcal{H}_{1}$ to the word written on a side of $\mathcal{B}$ that is farther from $p$. Thus the cyclic shift $u_{1}$ is equal in $\mathcal{H}_{1}$ to a word with fewer $k$-letters, contrary the assumption that $u$ is minimal.

Thus there are no two $k$-edges in $q$ connected by a $k$-band. Then, by part 3 , the number of $k$-edges on $q$ is bounded by a linear function in $|w|$. If $u$ contains a $k$-letter, we get a recursive bound for $n$. Hence we may suppose that $k$-letters do not occur in $u$.

5. The equality $w=u^{n}$ in $\mathcal{H}$ and the minimality of $w$ implies now that $w$ has no $k$-letters as well. Then, by OS2, Lemmas 10.4, 10.3], $\Delta$ has no hubs, and by OS2, Lemma 3.11], $\Delta$ has no $k$-annuli. So $\Delta$ has no $k$-edges at all. Therefore $w=u^{n}$ in $\mathcal{H}_{1}$. Now Lemma 3.11 OS2 implies that we may assume that $u$ contains no $\theta$-letters. Indeed, again if $u$ contains a $\theta$-letter and $n>|w|$, one of $\theta$-bands must connect two edges on the subpath labeled by a cyclic shift of $u$, contrary the assumption that $u$ is cyclically minimal. Since $w$ is minimal and $w=u^{n}$, the word $w$ has no $\theta$-letters too, and there are no $\theta$-edges in $\Delta$ by OS2, Lemma 3.11]. Finally, once again making use of the cyclic minimality of $u$ and the minimality of $w$, we conclude that $u$ and $w$ have no $a$-letters, and $\Delta$ has no $a$-bands for $a \notin \mathcal{A}\left(P_{1}\right)$. Since every $x$-cell must be a member of an $a$-band for $a \notin \mathcal{A}\left(P_{1}\right)$, all cells in $\Delta$ are $\mathcal{G}$-cells. It remains to use the solvability of the power problem in $\mathcal{G}$ (and in its free product with a free group generated by $x$-letters).

Proof of Theorem 1. Let $G$ be a group with solvable power problem. Using a sequence of HNN extensions as in Lemma 5, we can embed $G$ into a group $G_{1}$ with solvable power problem where every two elements of the same order are conjugate. Thus the conjugacy problem in $G_{1}$ is decidable. By Lemmas [10 and 11. $G_{1}$ can be embedded into a 2-generated group $G_{2}$ with solvable power and conjugacy problems. Then Lemma 12 allows us to embed $G_{2}$ into a finitely presented group $G_{3}$ with solvable power and conjugacy problems. Finally applying Lemmas 10 
and 11 again we embed $G_{3}$ into a 2-generated finitely presented (by Lemma 7) group $G_{4}$ with solvable power and conjugacy problems.

Theorem 3 follows immediately from Lemmas 8(6a), [10 and OS2, Theorem 1.1].

\section{References}

[Bel] Ilya Belyaev. A course paper, Moscow State University, 2003.

[Col1] Donald J. Collins. On embedding groups and the conjugacy problem. J. London Math. Soc. (2) 1 (1969) 674-682.

[Col2] Donald J. Collins. The word, power and order problems in finitely presented groups. Word problems: decision problems and the Burnside problem in group theory (Conf., Univ. California, Irvine, Calif., 1969; dedicated to Hanna Neumann), pp. 401-420. Studies in Logic and the Foundations of Math., Vol. 71, North-Holland, Amsterdam, 1973.

[KT] Kourovka Notebook. Unsolved Problems in Group Theory. 5th edition, Novosibirsk, 1976.

[LS] Roger Lyndon and Paul Schupp. Combinatorial group theory. Springer-Verlag, 1977.

[McC1] James McCool. Embedding theorems for countable groups. Canad. J. Math. 22 (1970), $827-835$.

[McC2] James McCool. Unsolvable problems in groups with solvable word problem. Canad. J. Math. 22 (1970), 836-838.

[O1] A.Yu. Olshanskii. SQ-universality of hyperbolic groups. Mat. Sb. 186 (1995), no. 8, 119132.

[O2] A. Yu. Ol'shanskii. On distortion of subgroups in finitely presented groups. Mat. Sb., 1997, V.188, N 11, 51-98.

[OS2] A.Yu. Olshanskii and M.V. Sapir. Conjugacy problem and Higman embeddings, accepted in Memoirs of AMS, 2003.

Alexander Yu. Ol'shanskii

Department of Mathematics

Vanderbilt University

alexander.olshanskiy@vanderbilt.edu

and

Department of Higher Algebra

MEHMAT

Moscow State University

olshan@shabol.math.msu.su
Mark V. Sapir

Department of Mathematics

Vanderbilt University

msapir@math.vanderbilt.edu 